\title{
THE INHIBITORY EFFECT OF GALANGIN ON CYTOKINES AND NITRIC OXIDE IN MICROGLIA BV2 CELL LINE
}

\author{
Nazeh M. Al-Abd ${ }^{1,3 *}$, Mustafa Kassim ${ }^{1}$, Asdren Zajmi ${ }^{2}$ \\ ${ }^{1}$ Department of Anesthesiology, Faculty of Medicine, University of Malaya, 50603 Kuala Lumpur, Malaysia \\ ${ }^{2}$ Department of Diagnostic and Allied Health Science, Faculty of Health and Life Sciences, Management \& \\ Science University, 40100, Shah Alam, Selangor, Malaysia. \\ ${ }_{3}^{3}$ Department of Para-Clinic, Faculty of Medicine and Health Science, University of Aden, Khormaksar, Aden, \\ Yemen. \\ *Corresponding Author: nazehali78@yahoo.com \\ Received: 6 Sept 2016 Revised manuscript received: 18 Nov 2017 \\ Accepted: 21 Nov 2017
}

\begin{abstract}
Galangin is an active flavonoid compound found in various plants, and is one of the primary constituents of honey and propolis. It has antioxidant, anti-inflammatory, antimicrobial and hepatoprotective properties. This study is intended to evaluate the effects of galangin on cytokines and nitric oxide (NO) secretion from microglia BV2 after being induced with lipopolysaccharide. Microglia BV2 was incubated with Galangin (with and without LPS) and dexamethasone for $24 \mathrm{~h}$. NO production in the cultured supernatant was measured with the Griess reaction. IL-6, IL-10, and TNF- $\alpha$ levels were measured using immunoassay. Results indicated that galangin did not exert cytotoxicity effect on the cell viability of BV2 macroglia at concentrations less than $40 \mu \mathrm{g} / \mathrm{mL}$. It was also observed that galangin had significantly suppressed the production of cytokines, such as IL-6, IL-10 and TNF- $\alpha$, as well as NO in LPS-induced BV2 cells. The antioxidant and anti-inflammatory properties of galangin are associated with its ability to inhibit $\mathrm{NO}$ and cytokines. Our results further showed the potential impact of galangin, thus suggesting that further development is feasible in order to leverage on its promising antioxidant and anti-inflammatory effects in treating neuroinflammatory diseases.
\end{abstract}

Key words: Galangin; Lipopolysaccharide (LPS); Nitric oxide (NO); Cytokines; Microglia BV2.

\section{INTRODUCTION}

Inflammation is an immune response against the changes in our cells or tissue due to external pathogens or damages. Various inflammatory processes are implicated in various diseases and these can lead to several pathological diseases such as cancer, diabetes, atherosclerosis and stroke, among others (Ferrero-Miliani et al., 2007; Kumar et al., 2014). Microglia are referred to as immune cells in the brain which are promptly activated in brain injury neurodegenerative processes; they secrete growth factors, inflammatory cytokines, reactive oxygen species (ROS) and others (Block \& Hong, 2005; Stolp \& 
Dziegielewska, 2009). Although the microglial activation is a compulsory function that is a part of host defence, the persistence of microglia activation is neurotoxic and it triggers neuronal cell death by the over-expression of proinflammatory mediators, which could in turn lead to neuro-inflammatory diseases (Wang et al., 2015). Many pharmacological studies have been conducted in pursuit of new agents to modulate the microglial activation and their production responses; this could stand prominently as an important therapeutic strategy to mitigate the effects of neuro-inflammatory diseases such as Alzheimer's disease, cerebral ischemia and Parkinson's disease (Block \& Hong, 2005; Stolp \& Dziegielewska, 2009). Microglia play a very important role in inflammatory diseases and neuropathology (Nelson et al., 2002). During the immune response caused by brain damage or neurodegenerative diseases, microglia will be activated to protect the brain from the changes and against infections. However, persistent activation of microglia and the secretion of neuro-inflammatory mediators such as cytokines and reactive oxygen species (ROS) which include nitric oxide and peroxynitrite as well as other free radicals can also prove deadly to tissue and to the host (Block et al., 2007). During inflammation, the role of macroglia is pivotal in phagocytosis and immunomodulation through the production of multiple kinds of pro-inflammatory cytokines and other inflammatory mediators indicated as markers of inflammation (Wang et al., 2015). Nonetheless, despite the important roles of pro-inflammatory cytokines in counteracting inflammation, their overproduction could lead to pathophysiological diseases (Valledor et al., 2010). Lipopolysaccharides (LPS) is wellestablished as important stimuli for activating macroglia in order to produce various cytokines and nitric oxide (NO). Neuro-inflammation produced by microglia comes in a variety of cytotoxic and proinflammatory factors due to the activated NF-kB and ROS. Excessive production of cytokines such as TNF- $\alpha$, IL- 6 and IL-10 as well as Nitric oxide (NO) can be further stimulated by inflammatory stimuli such as LPS treatment in the microglia, thus improving the immune response and subsequently lead to inflammation (Dantzer et al., 2008). However, although a low quantity of this neuro-inflammation is substantial due to its role in immune responses, its persistent release will bring about an uncontrolled accumulation of neuro-inflammation that could lead to the pathogenesis of several neurodegenerative diseases. The neuro-inflammation attenuation could potentially be a therapeutic or preventative approach in treating neurodegenerative diseases, including Alzheimer's disease and Parkinson's disease. LPS is the most prevalent inducer of neuro-inflammation in the brain. BV2 cell lines isolated from mouse are used widely in research pertaining to neurodegenerative disorders. Thus, identifying a way to reduce LPSinduced BV2 microglia inflammation would definitely facilitate neuroinflammation treatment. ROS play a significant role in inflammation through the activation of nuclear factor- $\kappa \mathrm{B}(\mathrm{NF}-\kappa \mathrm{B})$ and the monitoring of pro-inflammatory mediators. The release of $\mathrm{NO}$ is disadvantaged by the short half-life of the molecule and has a highly reactive free 
radical which exerts antimicrobial and antitumour effects as well as acts as a neurotransmitter. It is produced by inducible nitric oxide synthase (iNOS) from the oxidative L-arginine during inflammation; yet, the up-regulation of iNOS and $\mathrm{NO}$ is proportional with the pathogenesis of inflammatory diseases. Nonetheless, NO plays a vital role in the pathogenesis of multiple acute and chronic inflammatory diseases. NO produced by the macroglia has a significant function in the host defence response against various pathogens, damages and tumour cells. However, the over-production of $\mathrm{NO}$ is directly implicated by the development of cell damage, septic shock, various organ failures and neuropathological diseases with a putative mechanism that involves direct mitochondrial inhibition (O'Shea et al., 2002). Phenolic compounds are known to contain phenolic acid, flavonoids and other polyphenolic compounds. Plants appear to be the biggest source of these compounds, which are commonly found in flowers, leaves, stems and roots. The compounds have been shown to exert many pharmacological activities, including anti-inflammatory and antioxidant activities, free radical scavenging, immuneinhibition and stimulation, as well as possess anti-carcinogenic, antimicrobial, antiviral and neuroprotective qualities. Additionally, the compounds are potent enough to block or inhibit cellular pathways such as the NF-KB pathway as well as enzymes with phospholipase A2, xanthine oxidase and glutathione reductase (Kazlowska et al., 2010). Galangin is a natural flavonol belonging to the flavonoid class, which is a class of phenolic compounds, and is often found in honey, propolis, galangal, Helichrysumaureonitens and Alpiniaofficinarum. It is reported to have the ability to generate pharmacological activities such as anti-mutagenic, anticancer, radical scavenging, anti-oxidative, enzyme modulating and anti-inflammatory activities through the reduction of adhesion molecule and the inhibition of phospholipase A2 inhibition and antimicrobial activity (Afolayan \& Meyer, 1997; Ciolino \& Yeh, 1999; Cushnie \& Lamb, 2006; Duthie \& Morrice, 2012; Forbes et al., 2014; Meyer et al., 1997; Zhang et al., 2013). Our work aims to explore the potential of galangin in decreasing the immune response in BV2 microglia following LPS stimulation. In this study, we examined the effects of the galangin on the release of TNF- $\alpha$, IL- 6 , IL-10 and NO after the LPS-induction of the microglia BV2 cell line.

\section{MATERIALS AND METHODS}

\section{Reagents}

Dulbecco's Modified Eagle's Medium (DMEM), RPMI-1640 medium (RPMI), heat-inactivated foetal bovine serum (FBS), penicillin, streptomycin, phosphatebuffered saline (PBS, pH 7.4) and other tissue culture reagents were purchased from Invitrogen-Gibco (Grand Island, NY, USA). Galangin, griess, 3-(4,5-dimethylthizaol2yl)-2,5-diphenyl tetrazolium bromide (MTT) reagent and all other chemicals were purchased from Sigma-Aldrich (St. Louis, MO, USA). The cytokine assay kits used to determine cytokine concentration were purchased from R \& D (Minneapolis, MN, USA). The workflow of the study was depicted in Figure 1.

\section{Cell Culture and Viability}

BV2 mouse macroglia were purchased from Banca Biologica e Cell Factory, Genova, Italy. BV2 was cultured in Dulbecco's modified Eagle's medium (DMEM), 
supplemented with 10\% FBS containing $100 \mathrm{U} / \mathrm{mL}$ of penicillin/streptomycin, at $37^{\circ} \mathrm{C}$ in a $5 \% \mathrm{CO}_{2}$ humidified incubator. Cell viability was assessed using the MTT assay. Galangin was dissolved in dimethyl sulfoxide (DMSO), in order to obtain the required concentration $(5-40 \mu \mathrm{g} / \mathrm{mL})$, and the final concentration of DMSO in the culture medium was below $0.1 \%(\mathrm{v} / \mathrm{v})$.

In brief, cells were plated at a density of $1 x$ $10^{5}$ cells in a 96-well plate, and galangin was added to each plate at varying concentrations $(5-40 \mu \mathrm{g} / \mathrm{mL})$. After a $24 \mathrm{~h}$ incubation period, the absorbance was measured at $490 \mathrm{~nm}$. This assay was repeated thrice with triplicate samples in each measurement.

\section{Quantification of NO Production}

The BV2 cells were plated at $1 \times 10^{5}$ cells/well in 24-well plates and then incubated for $24 \mathrm{~h}$ with and without LPS $(1 \mu \mathrm{g} / \mathrm{mL})$, with and without galangin, and with $10 \mu \mathrm{M}$ of Dexamethasone. Nitrite levels in the culture media were ascertained using the Griess reaction assay and it was presumed to reflect NO levels, as described earlier (Kassim et al., 2010). In short, 100 $\mu \mathrm{L}$ of cell culture medium was mixed with $100 \mu \mathrm{L}$ of Griess reagent (equal volumes of $1 \%(\mathrm{w} / \mathrm{v})$ sulfanilamide in $5 \%(\mathrm{v} / \mathrm{v})$ phosphoric acid, and $0.1 \% \quad(\mathrm{w} / \mathrm{v})$ naphthylethylenediamine dihydrochloride) and incubated at room temperature for 15 min. The optical density was then determined at $540 \mathrm{~nm}$ in a microplate reader (Bio-Rad). The nitrite amount in the samples was measured with the serial dilution standard curve of sodium nitrite.

\section{Cytokine Assay}

Cytokines released from the treated BV2 macroglia were measured in cell culture supernatants using the commercial assay kits. Standard curves for each cytokine were generated using the kit-supplied reference cytokine samples. An evaluation was carried out on the production of the following cytokines: IL-6, IL-10, and TNF$\alpha$, as described by (Kassim et al., 2012).

\section{Statistical Analysis}

The results shown are summarized from three independent experiments, where they represent the mean \pm SEM. Significant differences were explored using a one-way ANOVA followed by the Dunett test with GraphPad Prism 5.0 software (GraphPad Software, San Diego, USA). $P$ values less than $0.05(p<0.05)$ were considered to be statistically significant.

\section{RESULTS}

\section{Effects of galangin on the cell viability of $B V 2$ cells}

The cytotoxicity of galangin on BV2 cells was tested using the MTT assay. The results indicated that galangin had no cellular toxicity on BV2 cells at a concentration of up to $40 \mu \mathrm{g} / \mathrm{mL}$, and this is illustrated in

Figure 2. Therefore, galangin at concentrations of and below $20 \mu \mathrm{g} / \mathrm{mL}$ was selected for the following experiments.

Effects of galangin on $\mathrm{NO}$ production in LPS-induced BV2

To study the anti-inflammatory effects of galangin, its effects on LPS-induced NO 
production was examined in this study. As made evident in Figure 2, in comparison to the control group, the production of NO did not show any increase in galangin-treated groups, whereas the production of NO had elevated drastically in the LPS-treated group. However, galangin groups and Dexamethasone suppressed the LPSinduced NO production in a dose-dependent manner. To assess the antineuroinflammatory effects of galangin on LPS-stimulated BV2 cells, proinflammatory mediators were measured with and without galangin at non-cytotoxic concentrations, ranging from 1.25 to 20 $\mu \mathrm{g} / \mathrm{mL}$. BV2 cells were pre-treated with galangin for $1 \mathrm{~h}$, and subsequently stimulated with LPS $(1 \mu \mathrm{g} / \mathrm{mL})$ for $24 \mathrm{~h}$. The LPS treatment had resulted in more than a 10-fold increase in the nitrite concentration in the culture media, compared to that of the untreated group. On the other hand, the pretreatment of the microglial cells with galangin for $1 \mathrm{~h}$ had caused a reduction in $\mathrm{NO}$ production implied by the nitrite concentration in LPSstimulated BV2 in a dose-dependent manner, as illustrated in Figure 3.

\section{Effects of galangin on cytokine production in LPS-induced BV2}

Cytokines, including TNF- $\alpha$, IL-6 \& IL-10, have been reported to play critical roles in inflammatory response. The effects of a 24 $\mathrm{h}$ treatment with different concentrations of galangin $(1.25-20 \mu \mathrm{g} / \mathrm{mL})$ on the cytokine production in LPS-activated BV2 macroglia are represented in Figure 4. Galangin significantly suppressed the production of the inflammatory mediators IL-6, IL-10, and TNF- $\alpha$ following the LPS induction in
BV2 macroglia cells in a dose-dependent manner. When the anti-inflammatory effect of galangin and that of Dexamethasone anti-inflammatory drugs were compared, it was observed that although Dexamethasone exhibited some inhibitory effects on the production of cytokines (including IL- 6 and IL-10) after the LPS induction in BV2, galangin at a concentration of $20 \mu \mathrm{g} / \mathrm{mL}$ inhibited LPS-induced cytokine production better and more successfully than Dexamethasone.

\section{DISCUSSION}

In cases of brain damage, there are many molecules that are released which automatically activate the microglia. However, microglial activation increased neuronal cell deaths in the brain by freeing cytotoxic agents such as cytokines, NO and others. Macroglia have a major role in immune responses in the brain; in the inflammatory process, they are involved in phagocytosis, releasing proinflammatory cytokines, NO and other mediators (Duque $\&$ Descoteaux, 2014). The present study shows that the anti-inflammatory effects of galangin in the activated BV2 microglia are stimulated by the LPS. Galangin significantly suppressed the microglial production of the inflammatory mediators of cytokines such as IL-6, IL-10 and TNF- $\alpha$ as well as NO in the LPS-induced BV2 macroglia in a dose-dependent manner. Increased levels of cytokines (such as TNF$\alpha$, IL-6 and IL-10) and NO often correlate with traumatizing brain injury, severe sepsis, several inflammatory autoimmune diseases and many others (Spite et al., 2009). Pro- and anti-inflammatory 
cytokines also have a significant role in the inflammatory response, and they are implicated in the development of chronic inflammatory diseases. As a result, the ongoing pro- and anti-inflammatory cytokine production stands to be the hallmark of various neurological diseases in the brain. Galangin significantly inhibits the generation of NO, IL- 6 , IL-10 and TNF- $\alpha$ in LPS-stimulated BV2 microglia in a concentration-dependent manner. One sure indication is that the inhibitory action of galangin on the production of inflammatory mediators takes place at the protein level. Some studies have reported on the benefits of natural compounds in neuro-protective and neuro-inflammatory health (Zhang et al., 2011). Several studies have delved into many natural compounds that could prevent neuronal deaths by means of up-regulating the expression of antiapoptotic proteins, glial-derived neurotrophic factor and the inhibition of inflammatory mediators in animal models. Furthermore, several preceding studies also highlighted the powerful antioxidant properties of galangin in vitro, as well as the strong radical scavenging and regulation of the inflammatory signalling through the ROS inhibition. Therefore, our results and others' suggest that galangin may stand as a useful antioxidant agent against ROS (Hewage et al., 2015). Previous studies illustrated that galangin reduces ear thickness, ulcers, dermal and epidermal thickness, epidermal hyperplasia and the infiltration of inflammatory cells (J. K. Choi \& S.-H. Kim, 2014). Treatment with of the skin lesions in atopic dermatitis (Zha et al., 2013). Galangin has an inhibitory effect on dermatitis development through the reduction of histamine following the decrease of edema; furthermore, galangin reduces both acute and chronic dermatitis (J. K. Choi \& S. H. Kim, 2014). Galangin also suppresses TNF- $\alpha /$ IFN-c-induced CCL17, IL-6, TNF- $\alpha$ and IL-1b keratinocytes as well as inhibits the gene expression of TNF- $\alpha$, IL- $1 b$ and IL- 6 in mast cells (J. K. Choi \& S. H. Kim, 2014; Madduma Hewage et al., 2015). In addition, previous studies also mentioned that the tropical application of galangin suppresses the development of dermatitis in in vitro and in vivo models. Galangin inhibits histamine release via the reduction of intracellular calcium of mast cells. It also inhibits the cytokine gene expression in mice arthritis model induced with collagen (Kim et al., 2013). Similarly, it significantly inhibits rheumatoid arthritis at both clinical and pathological levels (Huh et al., 2013). Its protective effect has been claimed to form a linkage with the down-regulation of IL-1b, TNF- $\alpha$, IL-17 and RANKL in the serum of experimental arthritis animals (Huh et al., 2013). Microglial cells are activated by extracellular stimuli, where the activation starts several crucial cellular responses, playing a role that is vital in the pathogenesis of neuro-inflammation. The various pro-inflammatory cytokines and neurotoxic mediators produced by the activated microglia are regarded as the contributing factors to neuronal injury and the pathogenesis of the neuro-inflammatory diseases. Thus, the control of the production of pro-inflammatory mediators could possibly be targeted at neuro-inflammationrelated diseases. Based on our data, galangin has demonstrated a remarkable ability to inhibit the production of cytokines and NO in LPS-stimulated BV2 microglial cells. The unnecessary production of 
cytokines and NO can be stimulated by inflammatory stimuli such as LPS-treatment in macroglia, thus increasing the immune response that can subsequently lead to inflammation (Dantzer et al., 2008). Hence, the inhibition of pro-inflammatory cytokines has been recognised as a target in anti-inflammatory treatments. Galangin has been shown to inhibit LPS-induced cytokine and NO production more successfully than Dexamethasone. One would have recognised that the resolution of inflammation is an active program regulated by temporal and special productions of specialized chemical mediators (Spite et al., 2009). In terms of pathogenic infection, the production of proinflammatory cytokines in immune cells results in acute or chronic inflammatory diseases (Yang et al., 2014). In the Nuclear factor-kappa B $(\mathrm{NF}-\kappa \mathrm{B})$, the principal transcription factor, which controls the inflammatory expression of multiple inflammatory and immune genes, such as cytokines, iNOS and COX, has a critical role in controlling inflammatory diseases. Earlier studies have shed light on the inhibitory effects of galangin on inflammatory cytokines due to the regulation of NF-kB pathway (Jung et al., 2014). Once the microglial cells are activated by a number of stimulations, the $\mathrm{I} \kappa \mathrm{B}$ protein is released and degraded, further causing free p50 and p65 which would subsequently translocate to the nucleus and lead to the production of cytokines, NO and others. Galangin has been discovered to impede the phosphorylation of I $\mathrm{B}$ and IKK in LPSstimulated macrophages (J. K. Choi \& S. H. Kim, 2014; Madduma Hewage et al., 2015). Furthermore, the inhibitory effect of galangin on the expression of proinflammatory cytokines and chemokines is mediated by means of blocking ERK, p38 MAPKs and NF-kB pathways in HaCaT cells (J. K. Choi \& S. H. Kim, 2014; Madduma Hewage et al., 2015). It also constricts collagen-induced arthritis by regulating NF-kB translocation and oxidative damage through the inhibition of NF-kB activation in rats fed with fructose (LI et al., 2008). Galangin inhibits the inflammatory cytokines through the regulation of caspase-1, NF-kB and MAPKs as well as through the prevention or treatment of mast cell-mediated allergic inflammatory disorders (Qu et al., 2017). It also functions as a modulator of inflammation and bone destruction in in vitro and in vivo (S. T. Chien et al., 2015; Huh et al., 2013). Furthermore, it suppresses RANKL-induced JNK, p38 MAPK and NF-kB activation by reducing phospho-c-Jun and NFATc1 expression and regulating $\mathrm{IkBa}$ activation in osteoclast precursors (S. T. Chien et al., 2015; Huh et al., 2013). Galangin has also been shown to significantly reduce the DNA-binding activity of NF- $\kappa \mathrm{B}$ in nuclear extracts taken from LPS-stimulated macrophages cells (S.T. Chien et al., 2015). Various biological effects of galangin such as antioxidant, antimicrobial, and anti-inflammatory effects have also been reported. Evidence showed galangin to specifically inhibit the production of TNF- $\alpha$ and ILs in whole blood cytokines in rat (Sivakumar \& Anuradha, 2011).

In a nutshell, the anti-inflammatory effects of (TNF- $\alpha$, IL-1,6 and 10) and the molecular mechanism involved in these effects are reported in this particular study 
and several others (J. K. Choi \& S.-H. Kim, 2014)

\section{CONCLUSION}

In summary, the present study has been able to show that galangin regulates neuroinflammatory activity through the suppression of NO, TNF- $\alpha$, IL-6 and IL-10 production in LPS-stimulated $\mathrm{BV} 2$ macroglia. The mechanism is made possible as the NF- $\mathrm{B}$ pathway is suppressed, particularly the activation of JNK and p38 in BV2 microglia cells. Further investigations are thus important to understand the molecular mechanisms at a transcriptional level as well as the transcription factors that control the inflammatory gene expression. It has been suggested in the data that galangin may well be regarded as a natural remedy to effectively prevent neuroinflammation, and as a promising pharmacological agent or food supplement. Earlier studies have illustrated the strong potential of galangin for therapeutic purposes against many inflammatory diseases.

\section{ACKNOWLEDGEMENTS}

This study was funded by the University Malaya Research Grants RG546-14HTM and RG313-14FR.
Figure 1: Workflow chart

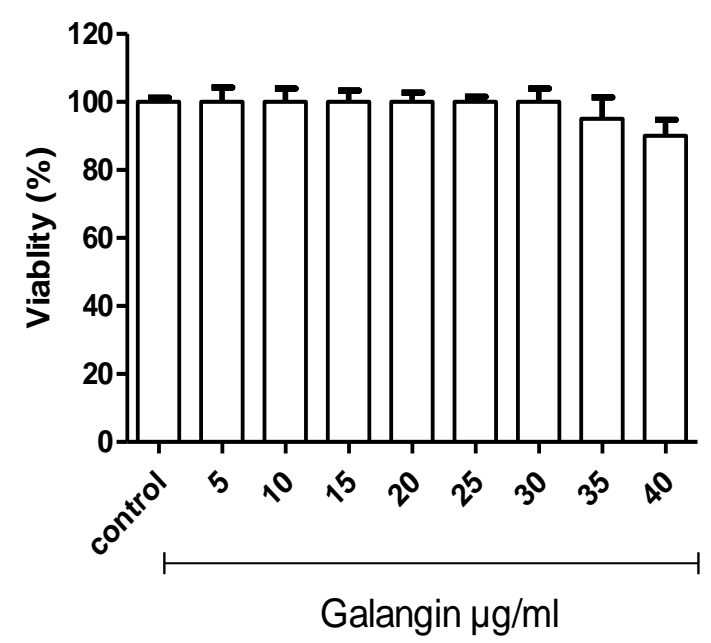

Figure 2: Cytotoxicity of galangin in BV2 cells. Cells were treated with increasing concentrations of galangin for 24 hours. Cell viability was assessed using the MTT assay. Mean \pm SD. $n=3$.

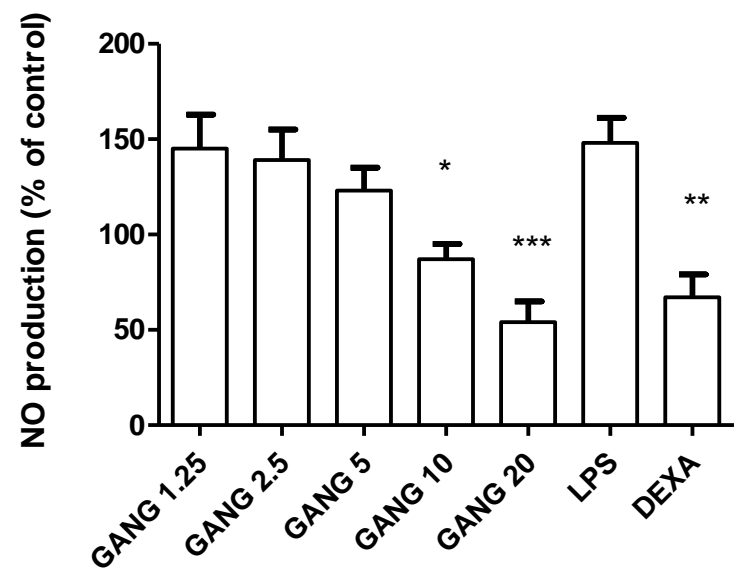

Figure 3: Effect of galangin on Nitric oxide (NO) production from BV2 stimulated with LPS in a dose-dependent manner. indicates significant change compared to control, $* p<0.01, * *$

$0.05 * * * p<0.005$. GANG $=$ Galangin, and DEXA= Dexamethasone 
A

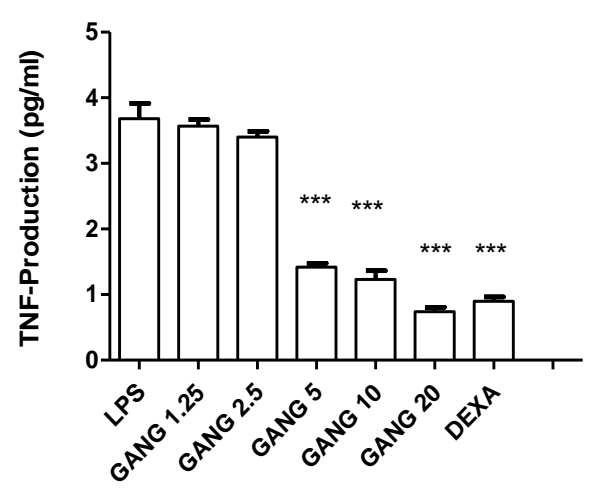

B

C
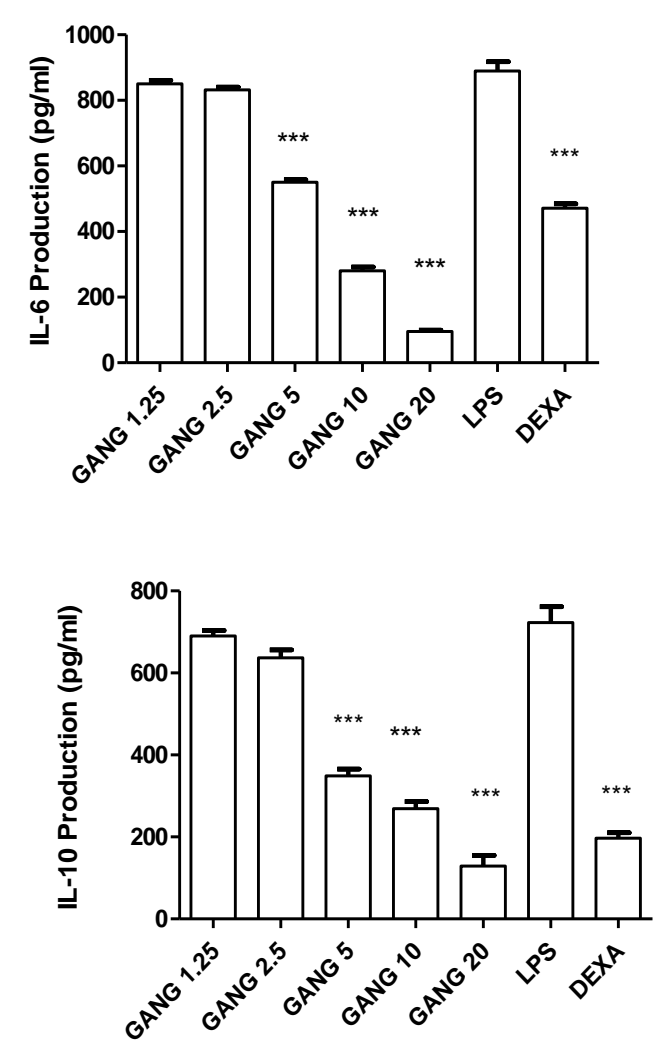

Figure 4: Effect of galangin on cytokines production from BV2 stimulated by LPS. (A) Galangin effect on the microglia production of the inflammatory mediators of TNF- $\alpha$ after LPS -induced BV2 macroglia in a dose-dependent manner. (B) Galangin effect on the microglia production of the inflammatory mediators of IL-6 after LPS -induced BV2 macroglia in a dosedependent manner. (C) Galangin effect on the microglia production of the inflammatory mediators of IL-10 after LPS -induced BV2 macroglia in a dosedependent manner. $* * * p<0.005$

\section{REFERENCES}

Afolayan, A., \& Meyer, J. (1997). The antimicrobial activity of $3,5,7-$ trihydroxyflavone isolated from the shoots of Helichrysum aureonitens. Journal of ethnopharmacology, 57(3), 177-181

Block, M. L., \& Hong, J. S. (2005). Microglia and inflammationmediated neurodegeneration: multiple triggers with a common mechanism. Prog Neurobiol, 76(2), 77-98.

Block M. L., Zecca, L., \& Hong, J. S. (2007). Microglia-mediated neurotoxicity: uncovering the molecular mechanisms. Nat Rev Neurosci, 8(1), 57-69.

Chien, S.-T., Shi, M.-D., Lee, Y.-C. TeC.C., \& Shih, Y.-W. (2015). Galangin, a novel dietary flavonoid, attenuates metastatic feature via PKC/ERK signaling pathway in TPA-treated liver cancer HepG2 cells. Cancer cell international, 15(1), 15.

Chien, S. T., Shi, M. D., Lee, Y. C., Te, C. C., \& Shih, Y. W. (2015). Galangin, a novel dietary flavonoid, attenuates metastatic feature via PKC/ERK signaling pathway in TPA-treated liver cancer HepG2 cells. Cancer Cell Int, 15, 15. 
Choi, J. K., \& Kim, S.-H. (2014). Inhibitory effect of galangin on atopic dermatitis-like skin lesions. Food and chemical toxicology, 68, 135-141

Choi, J. K., \& Kim, S. H. (2014). Inhibitory effect of galangin on atopic dermatitis-like skin lesions. Food Chem Toxicol, 68, 135-141

Ciolino, H., \& Yeh, G. (1999). The flavonoid galangin is an inhibitor of CYP1A1 activity and an agonist/antagonist of the aryl hydrocarbon receptor. British journal of cancer, 79(9-10), 1340.

Cushnie, T., \& Lamb, A. (2006). Assessment of the antibacterial activity of galangin against 4quinolone resistant strains of Staphylococcus aureus. Phytomedicine, 13(3), 187-191.

Dantzer, R., O'Connor, J. C., Freund, G. G., Johnson, R. W., \& Kelley, K. W. (2008). From inflammation to sickness and depression: when the immune system subjugates the brain. Nature reviews neuroscience, 9(1), 46-56.

Duque, G. A., \& Descoteaux, A. (2014). Macrophage cytokines: involvement in immunity and infectious diseases. Frontiers in immunology, 5.

Duthie, G., \& Morrice, P. (2012). Antioxidant capacity of flavonoids in hepatic microsomes is not reflected by antioxidant effects in vivo. Oxidative medicine and cellular longevity, 2012.

Ferrero- Miliani, L., Nielsen, O., Andersen, P., \& Girardin, S. (2007). Chronic inflammation: importance of NOD2 and NALP3 in interleukin- $1 \beta$ generation. Clinical \& Experimental Immunology, 147(2), 227-235.

Forbes, A. M., Lin, H., Meadows, G. G., \& Meier, G. P. (2014). Synthesis and anticancer activity of new flavonoid analogs and inconsistencies in assays related to proliferation and viability measurements. International journal of oncology, 45(2), 831-842.

Hewage, S. R. K. M., Piao, M. J., Kim, K. C., Cha, J. W., Han, X., Choi, Y. H., Chae, S., \& Hyun, J. W. (2015). Galangin (3, 5, 7-trihydroxyflavone) shields human keratinocytes from ultraviolet B-induced oxidative stress. Biomolecules \& therapeutics, 23(2), 165.

Huh, J. E., Jung, I. T., Choi, J., Baek, Y. H., Lee, J. D., Park, D. S., \& Choi, D. Y. (2013). The natural flavonoid galangin inhibits osteoclastic bone destruction and osteoclastogenesis by suppressing NF-kappaB in collagen-induced arthritis and bone marrow-derived macrophages. Eur J Pharmacol, 698(1-3), 57-66.

Jung, Y. C., Kim, M. E., Yoon, J. H., Park, P. R., Youn, H.-Y., Lee, H.-W., \& Lee, J. S. (2014). Anti-inflammatory effects of galangin on lipopolysaccharide-activated macrophages via ERK and NF- $\kappa \mathrm{B}$ pathway regulation. Immunopharmacology and immunotoxicology, 36(6), 426-432.

Kassim, M., Achoui, M., Mustafa, M. R., Mohd, M. A., \& Yusoff, K. M. (2010). Ellagic acid, phenolic acids, and flavonoids in Malaysian honey extracts demonstrate in vitro anti- 
inflammatory activity. Nutrition research, 30(9), 650-659.

Kassim, M., Yusoff, K. M., Ong, G., Sekaran, S., Yusof, M. Y. B. M., \& Mansor, M. (2012). Gelam honey inhibits lipopolysaccharide-induced endotoxemia in rats through the induction of heme oxygenase- 1 and the inhibition of cytokines, nitric oxide, and high-mobility group protein B1. Fitoterapia, 83(6), 1054-1059.

Kazlowska, K., Hsu, T., Hou, C. C., Yang, W. C., \& Tsai, G. J. (2010). Antiinflammatory properties of phenolic compounds and crude extract from Porphyra dentata. J Ethnopharmacol, 128(1), 123-130.

Kim, H. H., Bae, Y., \& Kim, S. H. (2013). Galangin attenuates mast cellmediated allergic inflammation. Food Chem Toxicol, 57, 209-216.

Kumar, V., Abbas, A. K., Fausto, N., \& Aster, J. C. (2014). Robbins and cotran pathologic basis of disease, Professional Edition: Expert Consult-Online: Elsevier Health Sciences.

LI, L., ZHONG, M., ZHANG, Y., \& ZHANG, W. (2008). Felodipine attenuates vascular inflammation in a fructose- induced rat model of metabolic syndrome via the inhibition of NF- $\kappa \mathrm{B}$ activation. Acta Pharmacologica Sinica, 29(9), 1051-1059.

Madduma Hewage, S. R., Piao, M. J., Kim, K. C., Cha, J. W., Han, X., Choi, Y. H., Chae, S., \& Hyun, J. W. (2015). Galangin (3,5,7-trihydroxyflavone) shields human keratinocytes from ultraviolet B-induced oxidative stress. Biomol Ther (Seoul), 23(2), 165-173.

Meyer, J., Afolayan, A., Taylor, M., \& Erasmus, D. (1997). Antiviral activity of galangin isolated from the aerial parts of Helichrysum aureonitens. Journal of ethnopharmacology, 56(2), 165169.

Nelson, P. T., Soma, L. A., \& Lavi, E. (2002). Microglia in diseases of the central nervous system. Ann Med, 34(7-8), 491-500.

O'Shea, J. J., Ma, A., \& Lipsky, P. (2002). Cytokines and autoimmunity. Nature Reviews Immunology, 2(1), 37-45.

Qu, J., Li, Y., Zhong, W., Gao, P., \& Hu, C. (2017). Recent developments in the role of reactive oxygen species in allergic asthma. Journal of thoracic disease, 9(1), E32.

Sivakumar, A. S., \& Anuradha, C. V. (2011). Effect of galangin supplementation on oxidative damage and inflammatory changes in fructose-fed rat liver. Chem Biol Interact, 193(2), 141-148.

Spite, M., Norling, L. V., Summers, L., Yang, R., Cooper, D., Petasis, N. A., Flower, R. J., Perretti, M., \& Serhan, C. N. (2009). Resolvin D2 is a potent regulator of leukocytes and controls microbial sepsis. Nature, 461(7268), 1287-1291

Stolp, H. B., \& Dziegielewska, K.M(2009). Review: Role of developmental inflammation and blood-brain barrier dysfunction in neurodevelopmental and neurodegenerative diseases. 
Neuropathol Appl Neurobiol, 35(2), 132-146.

Valledor, A. F., Comalada, M., Santamaría-Babi, L., Lloberas, J., \& Celada, A. (2010). 1 Macrophage Proinflammatory Activation and Deactivation: A Question of Balance. Advances in immunology, $108,1$.

Tang, W. Y., Tan, M. S., Yu, J. T., \&Tan, L. (2015). Role of pro-inflammatory cytokines released from microglia in Alzheimer's disease. Ann Transl Med, 3(10), 136.
Yang, J., Zhang, L., Yu, C., Yang, X.-F., \& Wang, H. (2014). Monocyte and macrophage differentiation: circulation inflammatory monocyte as biomarker for inflammatory diseases. Biomark Res, 2(1), 1.

Zha, W.-J., Qian, Y., Shen, Y., Du, Q., Chen, F.-F., Wu, Z.-Z., Li, X., \& Huang, M. (2013). Galangin abrogates ovalbumin-induced airway inflammation via negative regulation of NF-B. Evidence-Based Complementary and Alternative Medicine, 2013. 